\title{
A Polarizable Cationic Dummy Metal Ion Model
}

\author{
Ali Rahnamoun ${ }^{\ddagger \ddagger}$, Kurt A. O'Hearn ${ }^{\ddagger}$, Mehmet Cagri Kaymak ${ }^{\ddagger}$, Zhen Li†, \\ Kenneth M. Merz, Jr. †*, and Hasan Metin Aktulga** \\ tDepartment of Chemistry and Department of Biochemistry and Molecular Biology, Michigan \\ State University, 578 S. Shaw Lane, East Lansing, Michigan 48824-1322, United States \\ Department of Computer Science and Engineering, Michigan State University, 428 S. Shaw \\ Lane, East Lansing, Michigan 48824-1322, United States
}

\section{E-mail : rahnamou@msu.edu ; merz@chemistry.msu.edu ; hma@msu.edu}

\begin{abstract}
A novel locally polarizable multisite model based on the original cation dummy atom (CDA) model is described for molecular dynamics simulations of ions in condensed phases. Polarization effects are introduced by the electronegativity equalization model (EEM) method where charges on the metal ion and its dummy atoms can respond to the environment. This approach allows us to enhance the original fixed charge CDA model where the charge distribution can adapt to the local solvent structure. To illustrate the new $\mathrm{CDA}^{\mathrm{pol}}$ model, we examined properties of the $\mathrm{Zn}^{2+}$ ion in aqueous solution. Polarizable model and Lennard-Jones parameters were refined for an octahedrally coordinated $\mathrm{Zn}^{2+} \mathrm{CDA}$ to reproduce thermodynamic and geometrical properties. Using this locally polarizable model, we were able to obtain the experimental hydration free energy, ion-oxygen distance and coordination number coupled with the standard 12-6 Lennard-Jones model. This model can be used in myriad other applications where local polarization and charge transfer is important.
\end{abstract}


$\mathrm{M}$

etal ions play significant roles in biochemistry and materials science. Around one third of the

proteins in the protein databank contain metal ions ${ }^{1}$. Metal ions form complexes with surrounding amino acids and serve significant functional roles in biological systems ${ }^{2}$ including structural ${ }^{3}$, electron transfer and catalysis ${ }^{4}$. Modeling of systems including transition metals represent one of the more challenging tasks using standard classical simulations. The charge on transition metals is not constant and is affected by factors like the environment, nature of coordinated ligands and oxidation state of the metal. There is no general solution to date for the representation of transition metals in molecular force fields and it is still a matter of on-going development. The electronic cloud is usually distributed nonsymmetrically around the metal ion and can further change and redistribute in response to changes in the surrounding environment (Figure 1). Transition metals have $d$ and $f$ orbitals as their outermost orbitals which permit complicated chemical bonding patterns.
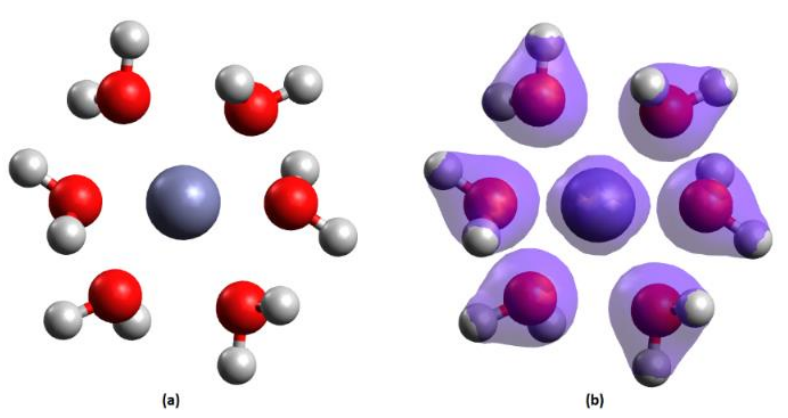

Figure 1. Polarizable models achieve higher fidelity than classical atom-centered fixed charge models through more accurate electrostatic interactions which leverage dynamically updated charge distributions. $\mathrm{Zn}^{2+}$ is coordinating with six water molecules in shown in (a) classical description and (b) with electron density distribution

Classical molecular dynamics (MD) simulations are mostly based on fixed atomic charge models that don't allow the electrostatic parameters to change as a function of geometry or respond to electrostatic potentials generated by nearby atoms. The simple idea of electrostatic interactions using fixed-point charges isn't entirely successful in many circumstances because the electrons are spread out in space and atomic charges change continuously. This has led to the creation of a range of polarizable force fields ${ }^{5,6}$. Properties of metal ions in aqueous solution are not well modeled by simple Lennard-Jones potentials which make the approximation that the ion has a formal charge $e^{7}$ Lennard-Jones potentials 
don't account for ion-induced dipole interactions, while fixed charge models don't account for polarization effects. Hence, further advances in modeling $d$ and f-block elements can significantly improve the performance of models for these ions.

Polarizable models with proper tuning can accurately predict charge distributions in molecular simulations, as such incorporation of polarization effects to traditional force fields is an ongoing effort ${ }^{5,6}$, 8-11. Polarizable force fields allow the electrostatic variables to change as a function of geometry or in response to the electrostatic potentials generated by nearby atoms and update the charges accordingly during a simulation. Applications of polarizable force fields to various problems such as protein-ligand binding $^{8}, \mathrm{pKa}$ prediction ${ }^{9}$ and ion solvation ${ }^{10}$ have been reported. Despite the benefits of polarizable models, they are computationally demanding due to their complexity. With continuing improvements in the speed of computational hardware, more accurate and consequently more computationally expensive molecular dynamics simulations can be afforded. Although quantum chemistry methods can be used to predict and update charges in systems of interest, QM methods are still limited to small systems simulated over shorter time scales only. The computational cost of the QM region also remains the rate limiting factor in hybrid quantum mechanics/molecular mechanics (QM/MM) simulations. In this regard, polarizable force fields offer significant improvements at the cost of an acceptable increase in the computational cost of a molecular simulation. In this paper, we utilize the recently developed hybrid ReaxFF/AMBER MD tool ${ }^{12}$, which introduces polarizability capabilities within the AMBER MD package (freely available in AMBERTools 21$)^{13}$ and allows us to use AMBER's thermodynamic integration (TI) capabilities for ReaxFF calculations. The ReaxFF/AMBER MD tool has been implemented by developing an efficient interface to the open source PuReMD Reactive MD program ${ }^{14,15}$ and uses the electronegativity equalization model (EEM) model within PUReMD for charge equilibration ${ }^{15}$. In EEM , one electronegativity and hardness parameter are assigned to each element and these parameters are optimized against QM-charge distributions. The energy is expressed as a function of the electronegativity and hardness parameters:

$$
E_{\text {elec }}=\left(\chi q+\frac{1}{2} \eta q^{2}\right)+E_{\text {Coulomb }}
$$

In Eq (1) for the self-energy contributions, $\chi$ represents the Mulliken electronegativity of an atom and $\eta$ represents twice the Parr-Pearson hardness. In the polarizable potential used in this study, Coulomb interaction with electrostatic shielding is adopted to prevent excessively high repulsion between nearby atoms as shown in Eq (2), where $C$ is the Coulomb constant in a vacuum, $q_{i}$ and $q_{j}$ denote the atomic 
charges, $r_{i j}$ is the distance between atoms $i$ and $j$, and $\gamma_{i j}$ is the electrostatic shielding between the types of atoms $\mathrm{i}$ and $\mathrm{j}$.

$$
E_{\text {Coulomb }}=C \cdot \frac{q_{i} q_{j}}{\left[r_{i j}^{3}+\left(\frac{1}{\gamma_{i j}}\right)^{3}\right]^{\frac{1}{3}}}
$$

The EEM model is based on atom-centered point charges, whereas a more realistic charge distribution should be smooth and anisotropic. To capture the anisotropic features, induced dipole models and/or off-center sites can be added to obtain a hybrid polarizable model ${ }^{16,17}$. The cationic dummy atom (CDA) model of Åqvist and Warshel ${ }^{18}$ is an attractive way to add multi-site charge distribution to a metal ion atom center. An octahedral geometry is the most common topological scheme for CDA simulations but other topological schemes like tetrahedral, tetrahedral-like with three dummy atoms and an empty site or pentagonal bipyramidal have also been used ${ }^{19,20}$. A tetrahedral CDA model with zinc as the central metal ion was used to evaluate the binding free energy of zinc to proteins and the free energy of binding contributed by the conformational fluctuations of the zinc-binding site was enhanced using this model compared to the standard, spherical ion model ${ }^{21}$. Pentagonal bipyramidal CDA models were used to improve the solvation, binding thermodynamics, and coordination geometries of $\mathrm{Ca}^{2+}$ and $\mathrm{Mg}^{2+}$ ions ${ }^{20}$. Octahedral CDA models have been used for enhancing the modeling of systems including $\mathrm{Mg}^{2+22}, \mathrm{Cu}^{2+23}$, $\mathrm{Mn}^{2+24}, \mathrm{Ni}^{2+25}$, and more ${ }^{26}$. While these models use standard 12-6 Lennard-Jones (LJ) potential and neglect polarization and ion-induced dipole interactions, attempts to develop a new type of $\amalg$ potential (12-6-4) by adding an additional $\frac{1}{r^{4}}$ term $^{27}$ to describe the ion induced dipole interactions for ions represented by standard single atom ${ }^{28}$ and ions represented by $\mathrm{CDA}^{29}$ have been reported.

In this work, we describe a novel CDA model, called CDA ${ }^{\mathrm{pol}}$, where polarization and ion-induced electrostatic interactions are explicitly incorporated using the fluctuating charge (FC) model. The CDA ${ }^{\text {pol }}$ model is very similar to the Drude model ${ }^{6,16}$ in spirit in which part of the mass and charge of the ion is fractioned into multiple sites anchored to the ion center. This model delocalizes a part of the total charge on dummy atoms placed around the ion center to mimic covalent bonds. To illustrate $\mathrm{CDA}^{\text {pol }}$, we report the results for an exemplar transition metal ion $\left(\mathrm{Zn}^{2+}\right)$ using both the standard CDA and the CDA ${ }^{\text {pol }}$ models. Six fractional positive charges were added to dummy atoms which are connected to the $\mathrm{Zn}^{2+}$ ion in an octahedral geometry at a constant distance (specifically, 0.9尺); the CDA interacts with one $U$ center on the central atom and seven (one middle ion and six dummy atoms) electrostatic centers on all CDA atoms. While reproducing properties of highly charged ions using fixed charge models has been 
challenging, we demonstrate that the novel $\mathrm{CDA}^{\mathrm{Pol}}$ model addresses many of these issues and outperforms the standard CDA model.

Separate electronegativity and hardness parameters were assigned to the middle ion and dummy atoms; these parameters were then optimized to allow the charges to fluctuate within the partial and total charge constraints specified in the $\mathrm{CDA}^{\mathrm{pol}}$ model. For modeling $\mathrm{CDA}^{\mathrm{pol}}$ solvation simulations, one or two different potentials were required depending on the method used (non-polarizable or polarizable). If the polarizable model is used, in addition to the AMBER potential, a separate potential is required for modeling the polarizable region. Consequently, two separate potential parameter optimizations were required. First, the polarizable potential parameters were tuned to reproduce the $\mathrm{CDA}^{\text {pol }}$ hydration by optimizing the middle ion and dummy atoms electrostatic energy parameters. Second, AMBER 12-6 $\mathrm{L}$ parameters were tuned to reproduce the target hydration free energies (HFE) and ion-oxygen distance (IOD) values (Figure 2). The details of the parameter optimizations procedures and training data for the model used in this work can be found in the supporting information. Using this locally polarizable model in combination with $\mathrm{TI}$ calculations, we can examine the effect of the CDA ${ }^{\text {pol }}$ model charge updates in response to environmental changes. To evaluate the fluctuations in the CDA charges, the charges on middle ion and dummy atoms were collected during a 50 ps equilibration of the solvated CDA ion. The charge fluctuation on the middle ion and dummy atoms and the deviations of charges on dummy atoms from the target value are shown in Figures S.3 and S.4 in the supporting information. These data show that the charges on these atoms place a slightly positive charge on the central ion (target was 0 charge and on average a charge of $\sim 0.019$ was observed during simulations) while the dummy atom charges fluctuate around the target value of +0.33 (Table 1 ).

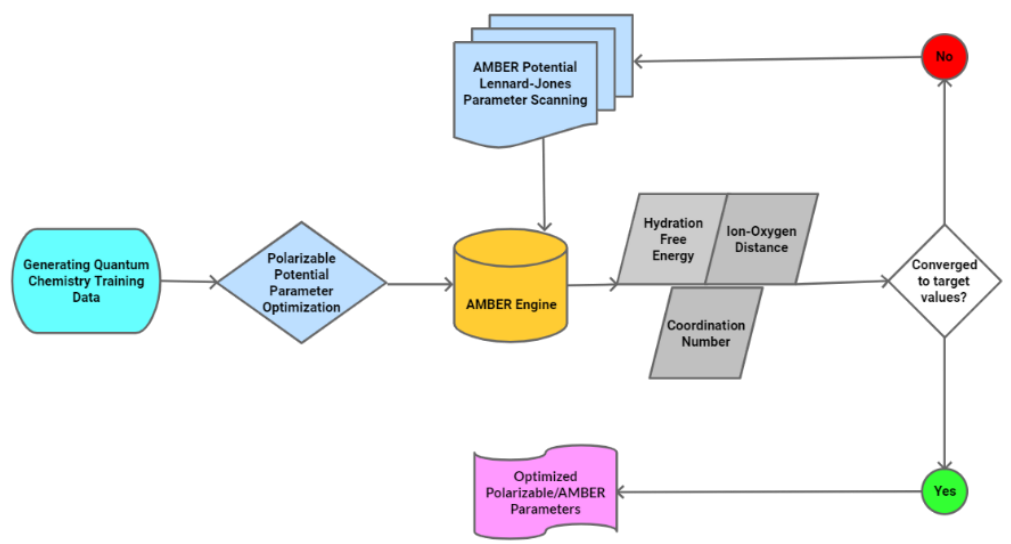

Figure 2. Flowchart of $\mathrm{CDA}_{\text {pol }}$ potentials training and modeling 
The dipole moment of the $\mathrm{CDA}^{\mathrm{pol}}$ model was calculated with respect to the molecule's center of mass as follows:

$$
\begin{gathered}
x_{c}=\left(\frac{1}{M}\right) \sum_{i=1}^{n}\left(m_{i} \times x_{i}\right) ; y_{c}=\left(\frac{1}{M}\right) \sum_{i=1}^{n}\left(m_{i} \times y_{i}\right) ; z_{c}=\left(\frac{1}{M}\right) \sum_{i=1}^{n}\left(m_{i} \times z_{i}\right) \\
P_{x}=\sum_{i=1}^{n}\left[q_{i} \times\left(x_{i}-x_{c}\right)\right] ; P_{y}=\sum_{i=1}^{n}\left[q_{i} \times\left(y_{i}-y_{c}\right)\right] \\
P_{z}=\sum_{i=1}^{n}\left[q_{i} \times\left(z_{i}-z_{c}\right)\right] \\
P=\sqrt{P_{x}^{2}+P_{y}^{2}+P_{z}^{2}}
\end{gathered}
$$

where $x_{i}, y_{i}$, and $z_{i}$ are atom coordinates, $m_{i}$ are the atomic masses, $M$ is the total mass of the molecule, $\left(\mathrm{x}_{c}, \mathrm{y}_{\mathrm{c}}, \mathrm{z}_{\mathrm{c}}\right)$ are the center of mass coordinates, $\mathrm{q}_{\mathrm{i}}$ are atom charges, and $\mathrm{P}$ is the dipole moment. The dipole moment fluctuations for $C^{2} A^{\text {pol }}$ during a 50 ps NPT equilibration is shown in Figure 3 . The average of these 1000 snapshot points is $0.22 \mathrm{D}$ which is not an unexpected value for the $\mathrm{CDA}^{\mathrm{pol}}$ model.

Table 1. Averages and standard deviations (SD) for $\mathrm{CDA}^{\mathrm{pol}}$ atomic charges during 50ps equilibration (1k data points).

\begin{tabular}{c|ccc} 
Atom & Target charge & Average charge & SD \\
\hline Zn & 0 & 0.0009 & 0.002 \\
D1 & 0.333 & 0.3321 & 0.016 \\
D2 & 0.333 & 0.3330 & 0.016 \\
D3 & 0.333 & 0.3330 & 0.017 \\
D4 & 0.333 & 0.3340 & 0.016 \\
D5 & 0.333 & 0.3328 & 0.016 \\
D6 & 0.333 & 0.3341 & 0.017
\end{tabular}




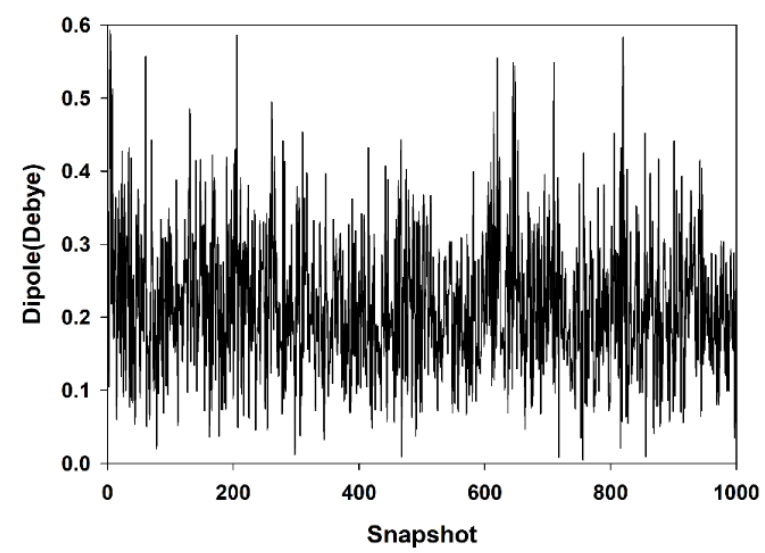

Figure 3. Instantaneous dipole moments of the $\mathrm{CDA}^{\text {pol }}$ molecule in solution during the simulation. The average value of the 1000 snapshots during $50 \mathrm{ps}$ is $0.25 \mathrm{D}$.

Three-point Gaussian integration of free energy difference of two states yielded the HFE shown in Figure 4. These contour graphs include the final HFE values in the $L$ scanning space using non-polarizable or polarizable models. When using the non-polarizable purely classical AMBER model, the HFE values vary from $-330 \mathrm{Kcal} / \mathrm{mol}$ to $-440 \mathrm{Kcal} / \mathrm{mol}$ in the $\amalg$ scanning space. However, when using the polarizable model, the HFE values vary from $-450 \mathrm{Kcal} / \mathrm{mol}$ to $-500 \mathrm{Kcal} / \mathrm{mol}$ in the $\mathrm{L}$ scanning space (note the different HFE scales in Figure 4). Hence, AMBER with fixed CDA charges is more sensitive to changes in $L$ parameters relative to the $C^{2}{ }^{\text {pol }}$ model where the charges fluctuate. IOD values were calculated from radial distribution functions (RDF) obtained with a grid resolution of $0.01 \AA$ based on the average volume of the trajectories in the range $0 \AA$ to $10.0 \AA$. The IOD values were obtained based on the protocol previously used for calculating the IOD values of highly charged metal ions ${ }^{27}$. The coordination number $(\mathrm{CN})$ values were determined via integrating the ion-oxygen RDF from the origin to its first minimum in the RDF plots. The resulting IOD values from both non-polarizable and polarizable models are shown in Figure 5.

HFE values represent a thermodynamic property, while IOD and CN represent structural properties. The experimental target HFE, IOD and $\mathrm{CN}$ for $\mathrm{Zn}^{2+}$ are $-467.3 \mathrm{Kcal} / \mathrm{mol}, 2.1 \AA$ and 6, respectively ${ }^{30}$. During $\mathrm{L}$ parameter scanning, the $\amalg$ parameter $\epsilon$ was varied from $1 \mathrm{kcal} / \mathrm{mol}$ to $3.4 \mathrm{kcal} / \mathrm{mol}$ in increments of 0.1 , while the $R_{\min } / 2$ parameter was scanned from $0.6 \AA$ to $1.0 \AA$ with a $0.1 \AA$ interval. Using the nonpolarizable model and the 12-6 $\mathrm{J}$ nonbonded model only, we couldn't reproduce the target HFE and IOD values simultaneously. However, the polarizable model using a 12-6 $\mathrm{L}$ potential reproduced the target HFE, IOD and CN values with good accuracy together at two different $\mathrm{L}$ parameter sets (see Table $\mathrm{S} .1$ in the supporting information) demonstrating the greater flexibility of $\mathrm{CDA}^{\mathrm{pol}}$. Overall, the best value 

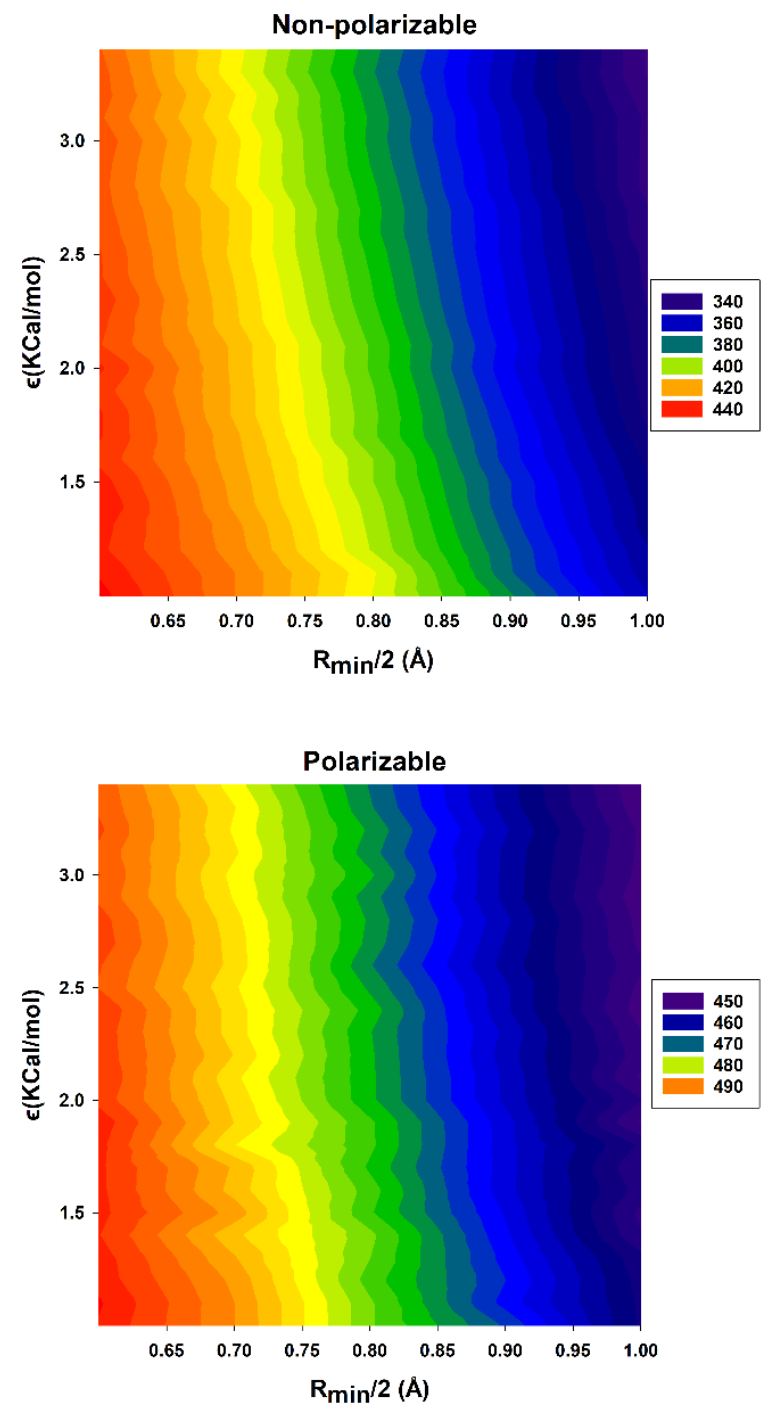

Figure 4. HFE values obtained from scanning $L$ parameters.

Absolute values are reported in figure legends.

is $R_{\min } / 2$ of $0.9 \AA$ and an $\varepsilon$ of $1.1 \mathrm{Kcal} / \mathrm{mol}$, which we have chosen as the default for the current model. This shows that with the local polarizability in $\mathrm{CDA}^{\mathrm{pol}}$, while the total charge of the $\mathrm{CDA}^{\mathrm{pol}}$ molecules is constrained to a constant value, the atomic charges respond to their environment yielding an instantaneous dipole that allows the thermodynamic and geometrical properties to be well reproduced using physically meaningful $\mathrm{U}$ parameters ${ }^{31}$. 

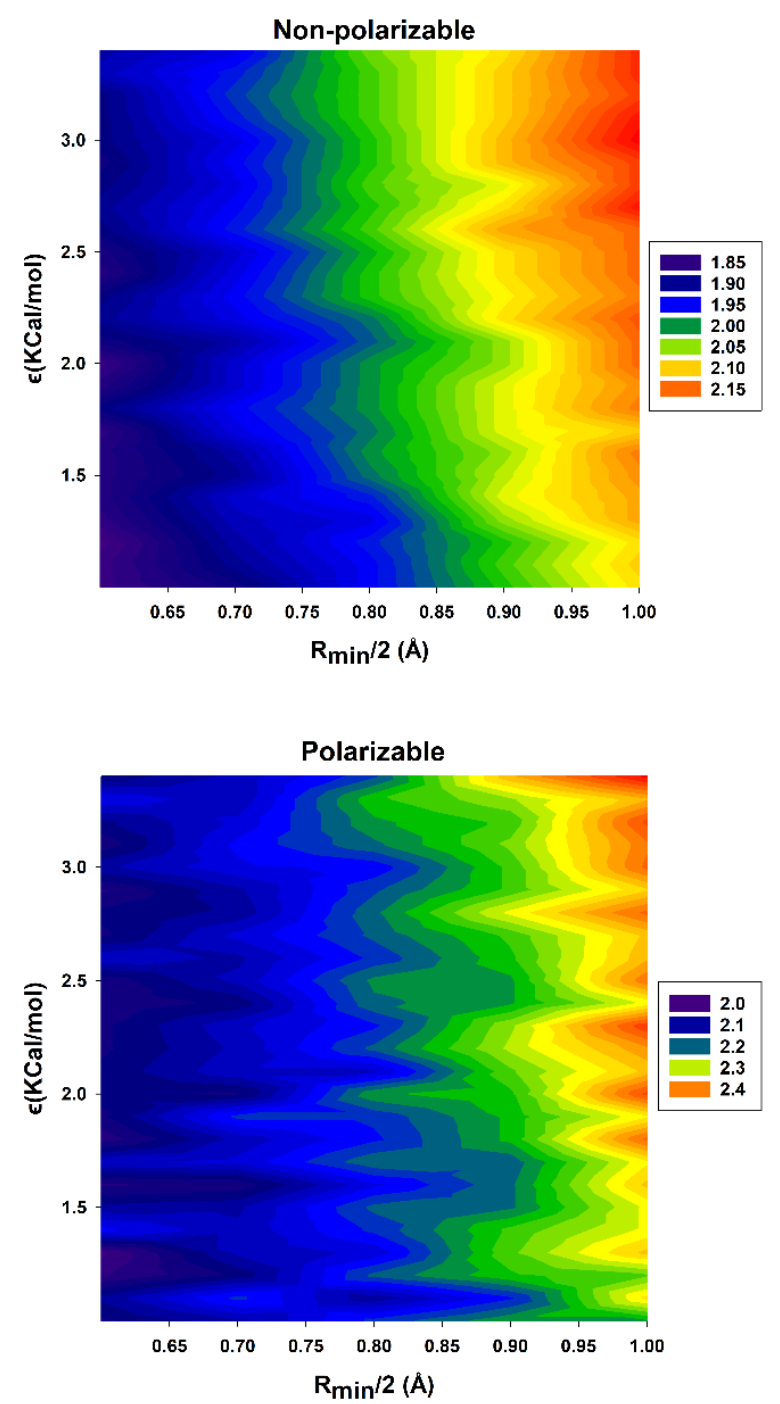

Figure 5. IOD values obtained from scanning $L$ parameters.

To obtain an estimate of the execution time of the simulations using non-polarizable or polarizable models, the average of the total simulation time of 500 NPT equilibration simulations using Simulated Annealing with NMR-Derived Energy Restraints (Sander) AMBER or Sander ReaxFF/AMBER of the solvated $\mathrm{CDA}^{\mathrm{pol}}$ were calculated. Each simulation was performed for 50 ps using a single Intel Xeon E52680v4 core. These benchmark calculations showed a 35\% increase in computational cost when using the $\mathrm{CDA}^{\mathrm{pol}}$ model. This shows that a modest increase in computational cost is sufficient to reap the benefits of a polarizable CDA model for more accurate calculations of thermodynamic and geometrical properties. 
In summary, a novel cation dummy atom model with polarization effects, called CDA ${ }^{\text {pol }}$, was described. $\mathrm{CDA}^{\text {pol }}$ includes polarization effects induced by a solvent surrounding highly-charged ions as well as including ion-induced dipole interactions which corresponds to non-symmetric charge distributions in the $\mathrm{CDA}^{\text {pol }}$ dummy atoms. In a proof-of-concept demonstration with the $\mathrm{CDA}^{\mathrm{pol}}$ model, experimental thermodynamic and geometrical observables for $\mathrm{Zn}^{2+}$ ion hydration were reproduced. The parameterization was performed through a scan of the 12-6 $\mathrm{L}$ potential and electrostatic parameters of the polarizable force field. The charges within the $\mathrm{CDA}^{\text {pol }}$ model were updated at every step with a constraint on the total charge of +2 . Our $\mathrm{CDA}^{\text {pol }}$ model showed less sensitivity to the changes in $L$ parameters which can be attributed to the inclusion of polarization and induced dipole effects in this model. It was demonstrated that this locally polarizable model reproduces the experimental HFE, IOD and $\mathrm{CN}$ simultaneously with a standard 12-6 $\mathrm{L}$ potential, while this was not observed using the classic CDA model under similar modeling conditions. Using the $\mathrm{CDA}^{\text {pol }}$ model for the simulation of ion hydration is advantageous over fixed charge CDA and conventional spherical models not only because it allows a greater flexibility in parameterization, but it is also able to better reproduce the thermodynamic and geometrical observables simultaneously. The ability to capture local environmental events in large systems using this polarizable model opens a range of problems that can be tackled using $\operatorname{CDA}^{\text {pol }}$ to address both chemical and biological processes.

\section{Acknowledgement}

This work was supported in part by a NIH grant (award number GM130641), and NSF CDS\&E grant (award number 1807622) and an NSF OAC grant (1835144). This work used computational resources provided by the Institute for Cyber-Enabled Research at Michigan State University.

\section{References}

(1) Peters, M. B.; Yang, Y.; Wang, B.; Fusti-Molnar, L.; Weaver, M. N.; Merz Jr, K. M. Structural survey of zinc-containing proteins and development of the zinc AMBER force field (ZAFF). J. Chem. Theory Comput 2010, 6 (9), 2935-2947.

(2) Tus, A.; Rakipović, A.; Peretin, G.; Tomić, S.; Šikić, M. BioMe: biologically relevant metals. Nucleic Acids Res 2012, 40 (W1), W352-W357.

(3) Holm, R. H.; Kennepohl, P.; Solomon, E. I. Structural and functional aspects of metal sites in biology. Chem. Rev 1996, 96 (7), 2239-2314.

(4) Andreini, C.; Bertini, I.; Cavallaro, G.; Holliday, G. L.; Thornton, J. M. Metal ions in biological catalysis: from enzyme databases to general principles. J. Biol. Inorg. Chem. 2008, 13 (8), 1205-1218.

(5) Baker, C. M. Polarizable force fields for molecular dynamics simulations of biomolecules. Wiley Interdiscip. Rev. Comput. Mol. Sci. 2015, 5 (2), 241-254. 
(6) Huang, J.; Lopes, P. E.; Roux, B.; MacKerell Jr, A. D. Recent advances in polarizable force fields for macromolecules: microsecond simulations of proteins using the classical Drude oscillator model. J. Phys. Chem. Lett 2014, 5 (18), 3144-3150.

(7) Li, P.; Merz Jr, K. M. Metal ion modeling using classical mechanics. Chem. Rev. 2017, 117 (3), 15641686.

(8) Jiao, D.; Golubkov, P. A.; Darden, T. A.; Ren, P. Calculation of protein-ligand binding free energy by using a polarizable potential. PNAS 2008, 105 (17), 6290-6295.

(9) MacDermaid, C. M.; Kaminski, G. A. Electrostatic polarization is crucial for reproducing pKa shifts of carboxylic residues in turkey ovomucoid third domain. J. Phys. Chem.B. 2007, 111 (30), 9036-9044.

(10) Grossfield, A.; Ren, P.; Ponder, J. W. Ion solvation thermodynamics from simulation with a polarizable force field. JACS 2003, 125 (50), 15671-15682.

(11) Cieplak, P.; Dupradeau, F.-Y.; Duan, Y.; Wang, J. Polarization effects in molecular mechanical force fields. J. Condens. Matter Phys. 2009, 21 (33), 333102. Ren, P.; Chun, J.; Thomas, D. G.; Schnieders, M. J.; Marucho, M.; Zhang, J.; Baker, N. A. Biomolecular electrostatics and solvation: a computational perspective. Quarterly reviews of biophysics 2012, 45 (4), 427-491.

(12) Rahnamoun, A.; Kaymak, M. C.; Manathunga, M.; Götz, A. W.; van Duin, A. C. T.; Merz, K. M.; Aktulga, H. M. ReaxFF/AMBER-A Framework for Hybrid Reactive/Nonreactive Force Field Molecular Dynamics Simulations. J. Chem. Theory Comput. 2020, 16 (12), 7645-7654. DOI: 10.1021/acs.jctc.0c00874.

(13) Case, D. A.; Aktulga, H. M.; Belfon, K.; Ben-Shalom, I.; Brozell, S. R.; Cerutti, D.; Cheatham III, T.; Cisneros, G.; Cruzeiro, V.; Darden, T. Amber 2021; University of California Press, 2021.

(14) Aktulga, H. M.; Fogarty, J. C.; Pandit, S. A.; Grama, A. Y. Parallel reactive molecular dynamics: Numerical methods and algorithmic techniques. Parallel Comput. 2012, 38 (4), 245-259.

(15) Aktulga, H. M.; Fogarty, J. C.; Pandit, S. A.; Grama, A. Y. Parallel reactive molecular dynamics: Numerical methods and algorithmic techniques. Parallel Comput. 2012, 38 (4-5), 245-259.

(16) Lamoureux, G.; Roux, B. Modeling induced polarization with classical Drude oscillators: Theory and molecular dynamics simulation algorithm. Chem. Phys. 2003, 119 (6), 3025-3039.

(17) Shi, Y.; Xia, Z.; Zhang, J.; Best, R.; Wu, C.; Ponder, J. W.; Ren, P. Polarizable atomic multipole-based AMOEBA force field for proteins. J. Chem. Theory Comput. 2013, 9 (9), 4046-4063.

(18) Åqvist, J.; Warshel, A. Free energy relationships in metalloenzyme-catalyzed reactions. Calculations of the effects of metal ion substitutions in staphylococcal nuclease. JACS 1990, 112 (8), 2860-2868.

(19) Tolbatov, I.; Marrone, A. Molecular dynamics simulation of the $\mathrm{Pb}$ (II) coordination in biological media via cationic dummy atom models. Theor. Chem. Acc. 2021, 140 (2), 1-12.

(20) Saxena, A.; Sept, D. Multisite ion models that improve coordination and free energy calculations in molecular dynamics simulations. J. Chem. Theory Comput. 2013, 9 (8), 3538-3542.

(21) Pang, Y.-P. Novel zinc protein molecular dynamics simulations: Steps toward antiangiogenesis for cancer treatment. J. Mol. Model. 1999, 5 (10), 196-202.

(22) Oelschlaeger, P.; Klahn, M.; Beard, W. A.; Wilson, S. H.; Warshel, A. Magnesium-cationic dummy atom molecules enhance representation of DNA polymerase $\beta$ in molecular dynamics simulations: Improved accuracy in studies of structural features and mutational effects. J. Mol. Biol. 2007, 366 (2), 687-701.

(23) Liao, Q.; Kamerlin, S. C. L.; Strodel, B. Development and application of a nonbonded Cu2+ model that includes the Jahn-Teller effect. J. Phys. Chem. Lett. 2015, 6 (13), 2657-2662.

(24) Wallin, C.; Kulkarni, Y. S.; Abelein, A.; Jarvet, J.; Liao, Q.; Strodel, B.; Olsson, L.; Luo, J.; Abrahams, J. P.; Sholts, S. B. Characterization of $\mathrm{Mn}$ (II) ion binding to the amyloid- $\beta$ peptide in Alzheimer $\mathrm{s}$ disease. J Trace Elem Med Biol. 2016, 38, 183-193. 
(25) Masetti, M.; Musiani, F.; Bernetti, M.; Falchi, F.; Cavalli, A.; Ciurli, S.; Recanatini, M. Development of a multisite model for $\mathrm{Ni}$ (II) ion in solution from thermodynamic and kinetic data. J. Comput. Chem. 2017, 38 (21), 1834-1843.

(26) Duarte, F.; Bauer, P.; Barrozo, A.; Amrein, B. A.; Purg, M.; Åqvist, J.; Kamerlin, S. C. L. Force field independent metal parameters using a nonbonded dummy model. J. Phys. Chem.B. 2014, 118 (16), 4351-4362.

(27) Li, P.; Song, L. F.; Merz Jr, K. M. Parameterization of highly charged metal ions using the 12-6-4 LJtype nonbonded model in explicit water. J. Phys. Chem.B 2015, 119 (3), 883-895.

(28) Li, P.; Merz Jr, K. M. Taking into account the ion-induced dipole interaction in the nonbonded model of ions. Journal of chemical theory and computation 2014, 10 (1), 289-297.

(29) Liao, Q.; Pabis, A.; Strodel, B.; Kamerlin, S. C. L. Extending the nonbonded cationic dummy model to account for ion-induced dipole interactions. J. Phys. Chem. Lett. 2017, 8 (21), 5408-5414.

(30) Marcus, Y. A simple empirical model describing the thermodynamics of hydration of ions of widely varying charges, sizes, and shapes. Biophys. Chem. 1994, 51 (2-3), 111-127.

(31) Li, P.; Roberts, B. P.; Chakravorty, D. K.; Merz Jr, K. M. Rational design of particle mesh Ewald compatible Lennard-Jones parameters for 2 metal cations in explicit solvent. J. Chem. Theory Comput. 2013, 9 (6), 2733-2748. 